\title{
The modeling of piezoelectrically excited waves in beams and layered substructures
}

\author{
Wolfgang Seemann ${ }^{\mathrm{a}, *}$, Alexander Ekhlakov ${ }^{\mathrm{a}}$, Evgeny Glushkov ${ }^{\mathrm{b}}$, \\ Natalia Glushkova ${ }^{b}$, Oleg Kvashab \\ ${ }^{a}$ Karlsruhe University, Karlsruhe, Germany \\ ${ }^{\mathrm{b}}$ Kuban State University, Krasnodar, Russian Federation
}

Received 4 January 2005; received in revised form 19 October 2006; accepted 24 October 2006

Available online 22 December 2006

\begin{abstract}
Two mathematical models for numerical simulation of traveling waves excited in an elastic waveguide by a pair of piezoceramic actuators are considered. The first model uses a simplified approach by Bernoulli-Euler beam theory and a simple actuation model. The second approach involves a boundary-value problem for an elastic layer with flexible piezoceramic strips bonded ideally to its surface. The limits of the applicability of the simplified beam model are then estimated by a comparison of the results both of the beam model and the solution of the patch-layer contact problem. Numerical results illustrate the effect of the actuators properties and position on the wave characteristics.
\end{abstract}

(C) 2006 Elsevier Ltd. All rights reserved.

\section{Introduction}

Nowadays piezoelectric elements are well used as actuators in many applications. The working principle of these components is based on the so-called direct and inverse piezoelectric effects which were discovered by Jacques and Pierre Curie in 1880. Though, the piezoelectric effect is thus known since the 19th century, wide spread use of piezoelectric ceramics began only in the second half of the 20th century.

The following groups of piezoelectric devices exist: generators, sensors, actuators, transducers, and combined systems. Piezoelectric ceramics are widely used in ultrasonic diagnostics in medicine, in ultrasonic non-destructive testing (NDT) of materials and constructions, in power piezoelectric ceramics - in ultrasonic welding, cleaning of surfaces, sputtering, drilling, etc. [1].

An important stage in the construction of a device is the development of adequate mathematical models to simulate its behavior. A correctly chosen model allows considerable reduction of the expenses spent for manufacturing and prototyping.

The present paper treats electro-elastic models simulating traveling waves excited in an elastic waveguide by thin flexible piezoceramic patches oscillating under the action of a harmonic electric field. The simplest model

\footnotetext{
*Corresponding author.

E-mail addresses: seemann@itm.uni-karlsruhe.de (W. Seemann), evg@math.kubsu.ru (E. Glushkov).
} 
uses the Euler-Bernoulli beam theory to describe the bending waves while the action of actuators is simulated by time harmonic moments applied to the points of the patches edges.

Due to its simplicity this model allows us to derive an explicit analytical expression for the waves generated by a pair of actuators, which is convenient for frequency analysis of the system characteristics depending on the size and location of the actuators, and on the electromechanical properties. The limits of applicability of this model are estimated by a comparison with the results obtained with a more complex elastic layer model based on a semi-analytical solution of the Wiener-Hopf integral equation to which the patch-layer contact problem is reduced.

\section{General statement}

Piezoceramic actuators for the generation of traveling waves are used in form of piezoceramic elements bonded to the surface of waveguides (Fig. 1). Their ability to excite waves is based on the property to respond to an electric field with mechanical strain. The strain is caused by bulk forces induced in the ceramics. For low electric fields they are proportional to the applied voltage. The polarization of the patch is in thickness direction and the electrodes are located on the bottom and top surface of the patch. Therefore, a voltage applied to the electrodes leads to an electric field $\mathbf{E}$ also in thickness direction.

A detailed description of the constitutive equations for the piezoelectric effect in solids may be found in Refs. [2,3]. For a thin flexible patch subjected to a transverse electric field $\mathbf{E}=\left(0,0, E_{z}\right)$ and having only a normal stress $\sigma_{x}$ in $x$-direction we assume that stress components $\sigma_{x z}, \sigma_{y z}, \sigma_{z}$ as well as strain components $\gamma_{x z}$, $\gamma_{y z}$ are negligible. Within the plane-strain state supposed the general Hook's law is reduced to the following form:

$$
\sigma_{x}=\frac{E_{0}}{1-v_{0}^{2}}\left(\varepsilon_{x}-d_{31}\left(1+v_{0}\right) E_{z}\right) .
$$

Here $\sigma_{x}, \varepsilon_{x}$ are the components of the stress and strain tensors in $x$-direction, $E_{0}$ is Young's modulus, $v_{0}$ is Poisson's ratio of the patch, $E_{z}$ is the strength of the electric field, and $d_{31}$ is a piezoelectric constant connecting electric field and mechanical strain in the patch material. Only this component of the electromechanical coupling tensor is important in this case.

An elementary volume of a bonded patch of infinitesimal length $\mathrm{d} x$, height $h_{0}$ and width $b_{0}$ (Fig. 2) is therefore subjected to the total lengthwise force

$$
\mathrm{d} f_{x}=b_{0} h_{0} \mathrm{~d} \sigma_{x}-q b_{0} \mathrm{~d} x-\ddot{v} \mathrm{~d} m .
$$

It results from the force proportional to the area of the cross-section $b_{0} h_{0}$ due to the change of $\sigma_{x}$ within $\mathrm{d} x$ and a force due to the contact shear stress $\tau_{x z}=q$ applied to the contact area $b_{0} \mathrm{~d} x$. Finally, there is the inertia force which is proportional to the acceleration $\ddot{v}$ and to the mass $\mathrm{d} m=\rho_{0} b_{0} h_{0} \mathrm{~d} x$ of the elementary volume; $\rho_{0}$ is the patch density, and $v$ is the displacement of the center of mass in $x$-direction.

The equilibrium condition $\mathrm{d} f_{x}=0$ implies the equilibrium equation:

$$
\frac{\mathrm{d} \sigma_{x}}{\mathrm{~d} x}-\frac{1}{h_{0}} q-\rho_{0} \ddot{v}=0 .
$$

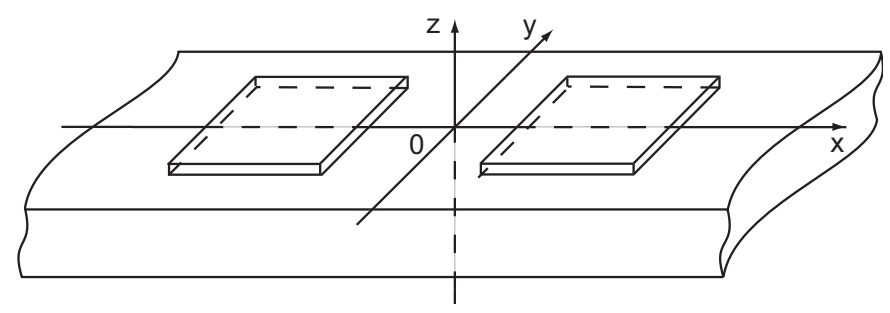

Fig. 1. Waveguide with piezoelectric patches. 


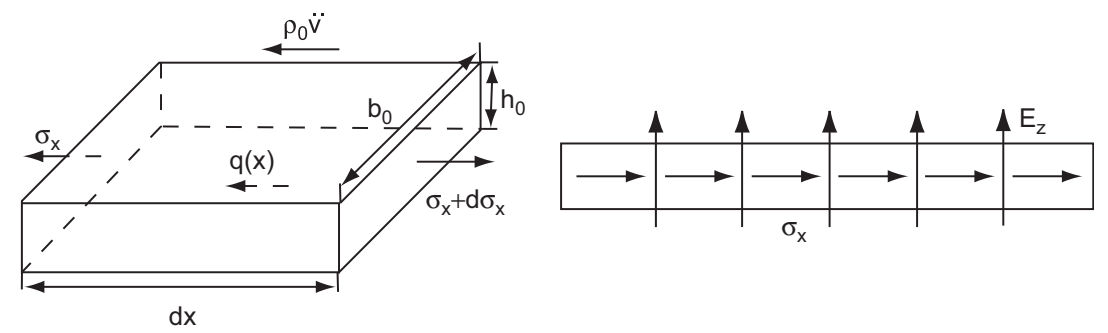

Fig. 2. Forces acting on an infinitesimal volume of the piezoceramic patch.

With continuous dependence of the displacement $v(x, t)$ on $x$, the elongation $\varepsilon_{x}=\mathrm{d} v / \mathrm{d} x$, so that Eq. (3) yields a $1 \mathrm{D}$ equation of motion of a thin piezoceramic patch bonded to a plane surface:

$$
\frac{E_{0}}{1-v_{0}^{2}} \frac{\partial^{2} v}{\partial x^{2}}-\rho_{0} \frac{\partial^{2} v}{\partial t^{2}}=\frac{1}{h_{0}} q .
$$

Furthermore, we assume that within the patch all fields are independent of $y$ and $z$, while, in addition, $E_{z}$ is constant: $\mathrm{d} E_{z} / \mathrm{d} x=0$. With this assumption the displacement $\mathbf{u}$ of the waveguide should also be independent of $y$. Therefore, in this paper we will restrict ourselves to a 2D plane-strain model with strip actuators and inplane vectors of motion $\mathbf{u}=\left(u_{x}, u_{z}\right)$.

As is well-known, any arbitrary transient motion, including the patch displacement $v(x, t)$, can be deduced in terms of steady-state harmonic oscillations $v(x, \omega) \mathrm{e}^{-\mathrm{i} \omega t}$ :

$$
v(x, t)=\frac{1}{\pi} \operatorname{Re} \int_{0}^{\infty} v(x, \omega) \mathrm{e}^{-\mathrm{i} \omega t} \mathrm{~d} \omega .
$$

With respect to the complex amplitude $v(x, \omega)$ the equation of motion takes the form of a $1 \mathrm{D}$ wave equation:

$$
\frac{\mathrm{d}^{2} v}{\mathrm{~d} x^{2}}+\kappa_{0}^{2} v=q_{0}
$$

with $\kappa_{0}^{2}=\omega^{2} \rho_{0}\left(1-v_{0}^{2}\right) / E_{0}$ and $q_{0}=q\left(1-v_{0}^{2}\right) /\left(h_{0} E_{0}\right)$.

In this way, a solution of a harmonic steady-state problem can be treated as an intermediate step providing a frequency spectrum for a general transient problem. As usual, in the harmonic statement all electromechanical fields are deemed to be expressed via their complex amplitudes (spectrums): $\mathbf{u}(x, \omega) \mathrm{e}^{-\mathrm{i} \omega t}$, $\sigma_{x}(\omega) \mathrm{e}^{-\mathrm{i} \omega t}, E_{z}(\omega) \mathrm{e}^{-\mathrm{i} \omega t}$, and so on. Due to the linearity of the problem the harmonic factor $\mathrm{e}^{-\mathrm{i} \omega t}$ is omitted in Eq. (6) and henceforth.

Whenever non-confusing, the dependence on $\omega$ in the complex amplitudes is usually omitted as well. Absolute values of the complex amplitudes give amplitudes of the time-harmonic oscillations described, while their arguments relate to the phase shifts. For example, in an induced electric field $E_{z}=\left|E_{z}\right| \mathrm{e}^{\mathrm{i} \varphi},\left|E_{z}\right|$ is its amplitude and $\varphi$ is the phase shift in the time dependence $\mathrm{e}^{-\mathrm{i}(\omega t-\varphi)}$.

Let the actuator be in contact with the base in a domain $\Omega:|x| \leqslant a$. If its profile edges are stress-free: $\left.\sigma_{x}\right|_{x= \pm a}=0$, we arrive at the two-point boundary condition:

$$
\mathrm{d} v /\left.\mathrm{d} x\right|_{x= \pm a}=e_{0}\left(1+v_{0}\right), \quad e_{0}=d_{31} E_{z} .
$$

Nevertheless, the ODE problem (4)-(7) is not closed yet, because there are two unknown functions $v$ and $q$ coupled in one equation (4). To close the problem, some relation between $v$ and $q$ following from the elastic properties of the base must be taken into account in addition to Eq. (4).

With any layered elastic substructure (laminated composite, stratified half-space, and so on) the required relation can be derived in terms of Green's matrix $\mathbf{k}(x)$. Generally, the displacements at a point of an elastic substructure, caused by a surface load $\mathbf{q}$ applied into a domain $\Omega$, can be represented as a convolution of $\mathbf{k}$ 
with $\mathbf{q}$ (e.g. see Ref. [4]). For an in-plane motion it takes the following form:

$$
\mathbf{u}(\mathbf{x})=\int_{\Omega} \mathbf{k}(\mathbf{x}-\xi) \mathbf{q}(\xi) \mathrm{d} \xi, \quad \mathbf{x}=(x, z), \quad \xi=(\xi, 0)
$$

with $2 \times 2$ matrix k, which in turn can be expressed through its Fourier symbol

$$
\mathbf{K}(\alpha, z)=\mathscr{F}[\mathbf{k}] \equiv \int_{-\infty}^{\infty} \mathbf{k}(x, z) \mathrm{e}^{\mathrm{i} \alpha x} \mathrm{~d} x
$$

by $\mathscr{F}$ we denote the Fourier transform operator. It leads to the alternative representation

$$
\mathbf{u}(\mathbf{x})=\frac{1}{2 \pi} \int_{\Gamma} \mathbf{K}(\alpha, z) \mathbf{Q}(\alpha) \mathrm{e}^{-\mathrm{i} \alpha x} \mathrm{~d} \alpha
$$

in which

$$
\mathbf{Q}=\mathscr{F}[\mathbf{q}] \equiv \int_{\Omega} \mathbf{q}(\xi) \mathrm{e}^{\mathrm{i} \alpha \xi} \mathrm{d} \xi
$$

is the Fourier symbol of the load q. The integration path $\Gamma$ goes in the complex plane $\alpha$ along the real axis $\operatorname{Im} \alpha=0$, deviating from it only to round the real poles and branch points of the $\mathbf{K}$ components. The direction of deviation is fixed in accordance with the principle of limiting absorption.

In the case considered, a flexible patch produces only shear load $\mathbf{q}=(q, 0)$, so that only the first column of $\mathbf{k}$ works in Refs. (8) and (9). Finally, the equality

$$
\left.u_{x}\right|_{z=0}=v, \quad x \in[-a, a], \quad z=0,
$$

connecting the patch and surface displacements in the contact domain $\Omega$, provides an additional relation between $v$ and $q$ required for closing the problem:

$$
v(x)=\int_{\Omega} k_{11}(x-\xi) q(\xi) \mathrm{d} \xi \equiv \mathscr{K} q, \quad x \in \Omega .
$$

Here $k_{11}(x)$ is the $(1,1)$ entry of the matrix $\mathbf{k}(\mathbf{x})$ taken at $z=0$.

By this means, in the general statement one has to find unknown functions $v$ and $q$ from the integrodifferential boundary value problem (4), (7), and (11). The electric field $E_{z}$, entering into the boundary condition via $e_{0}$, is a source of motion. The solution depends also on the electromechanical properties of the patch, described by the constants of Eqs. (1), (4) and on the elastic properties of the substructure, incorporated in the integral operator $\mathscr{K} q$. It should be noted that the motion of an elastic base excited by the actuator are described explicitly by the integrals (8), (9) as soon as $q$ has been calculated. In particular, physically evident representation of traveling waves, which we seek for, are derived from the path integral (9) as a contribution of residuals for the real poles $\zeta_{k}$ of $\mathbf{K}(\alpha, z)$ components:

$$
\mathbf{u}(x, z) \sim \sum_{k=1}^{N} \mathbf{a}_{k}^{ \pm}(z) \mathrm{e}^{ \pm \mathrm{i} \zeta_{k} x}, \quad x \rightarrow \pm \infty,
$$

$\mathbf{a}_{k}^{\mp}=\mp \mathrm{i}$ res $\left.\mathbf{K}(\alpha, z)\right|_{\alpha=\mp \zeta_{k}} \mathbf{Q}\left(\mp \zeta_{k}\right) ; N$ is a number of the real poles $\zeta_{k}$.

Finally, it is worth to note, that the integral representations (8) and (9) are quite applicable in the case of several actuators too. In this case the contact domain consists of $M$ disconnected subdomains $\Omega_{j}: \Omega=\bigcup_{j=1}^{M} \Omega_{j}$. In other respects all calculations remain as above.

We start, however, from a simplified beam model of a substructure.

\section{Beam model}

Let us consider a straight elastic beam of rectangular cross-section $b \times h(b$ is a width and $h$ is a height (Fig. $3)$ ) with stress-free sides. The beam length $L$ is much more than $b$ and $h$, so that in theoretical models the beam is supposed to be infinite in $x$ direction. Thus, in a Cartesian coordinate system $\mathbf{x}=(x, y, z)$ the beam occupies a volume $|x|<\infty,|y| \leqslant b / 2,|z| \leqslant h / 2$. 


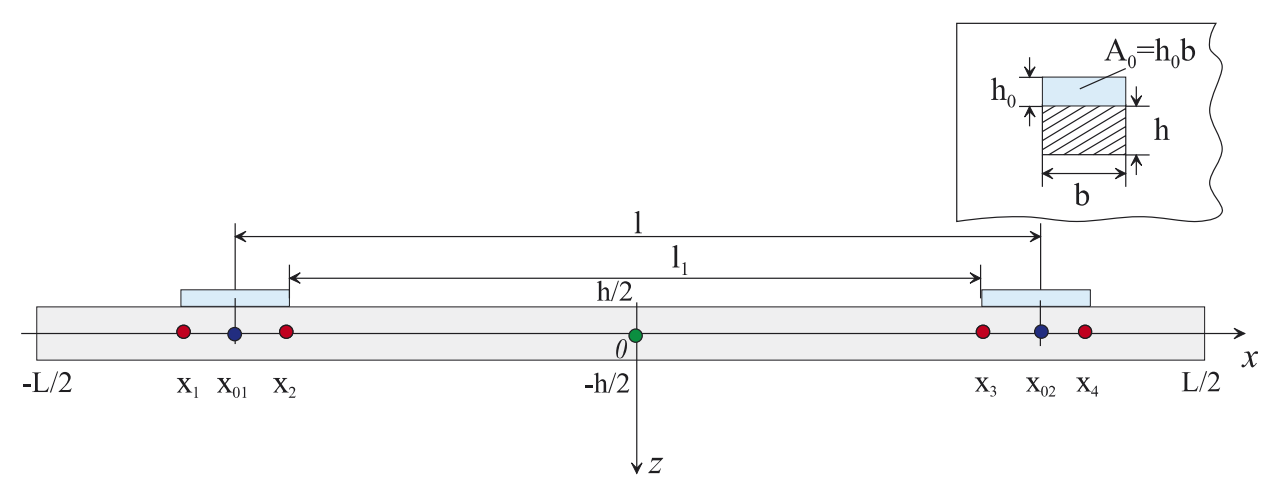

Fig. 3. Beam geometry.

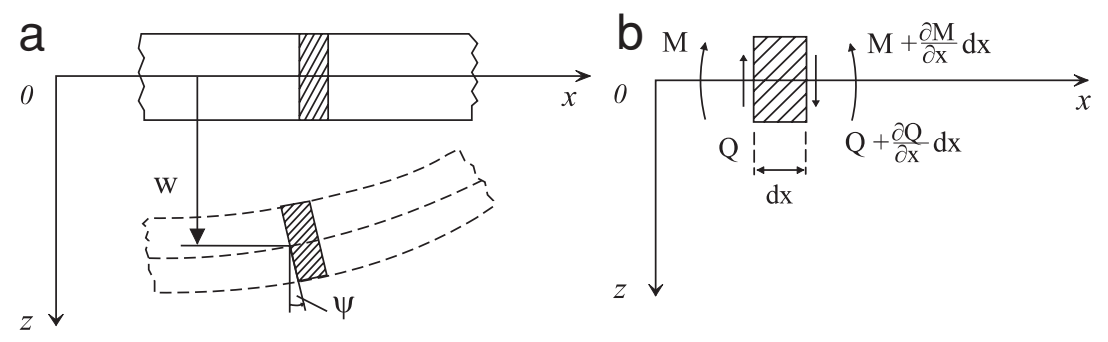

Fig. 4. Displacement and rotation of a beam element.

Transverse displacements $w(x, t)$ of the middle line $(y=0, z=0)$ are small in comparison with the height $h$, they comply with the Euler-Bernoulli beam theory [5].

Let us consider bending waves in the beam generated by tangential displacements $v_{n}, n=1,2$ of two thin piezoceramic patches fixed at the beam's surface $z=h / 2$ at a distance $l$ between their centers $x_{0,1}=-l / 2$ and $x_{0,2}=l / 2$ (Fig. 3). The motion is excited by transverse electric fields $E_{z, n}=\left|E_{z, n}\right| \mathrm{e}^{\mathrm{i} \varphi_{n}}, n=1,2$, applied to the patches. Here $\varphi_{n}$ are the phase shifts of the harmonic oscillations of the actuators. Therefore, there may be a temporal phase shift between the excitation of the two patches.

With ideal bonding and small film thickness $\left(h_{0} \ll h\right)$ the shear contact stress $q(x)$ between the ceramics and the beam can be approximated by $\delta$-distributions given at the limiting points $x_{j}, j=1, \ldots, 4$ of the contact intervals: $x_{1} \leqslant x \leqslant x_{2}$ and $x_{3} \leqslant x \leqslant x_{4}$ for the first and the second patches, respectively. This has the same effect as application of discrete bending moments [6]

$$
M_{j}=E_{0} A_{0} \frac{h}{2} d_{31} E_{z, n}, \quad j=1, \ldots, 4, n=[(j+1) / 2]
$$

to the left ( $j$ is odd) and to the right (even) ends of the $n$th actuator interval.

For a correct use of these moments together with the equations of motion (17) or (18) let us consider their derivation in more detail. An elementary part of the beam of a small length $\mathrm{d} x$ is subjected to the total lengthwise force (Fig. 4)

$$
\mathrm{d} f_{x}=\left(Q+\frac{\partial Q}{\partial x} \mathrm{~d} x\right)-Q-\rho A \frac{\partial^{2} w}{\partial t^{2}} \mathrm{~d} x,
$$

where $Q$ is a shear force, $M$ is a bending moment and $\psi$ is an angle of rotation. Eq. (17) comes from the equilibrium condition $\mathrm{d} f_{x}=0$, which implies

$$
\frac{\partial Q}{\partial x}=\rho A \frac{\partial^{2} w}{\partial t^{2}} .
$$


According to the Euler-Bernoulli beam theory (see Eqs. (4.53) in Ref. [5]) $Q, M, \psi$ and $w$ are connected by the relations

$$
Q=\frac{\partial M}{\partial x}, \quad M=E I \frac{\partial \psi}{\partial x}, \quad \psi=-\frac{\partial w}{\partial x} .
$$

Their substitution into Eq. (15) results in equation of motion [5]:

$$
E I \frac{\partial^{4} w}{\partial x^{4}}+\rho A \frac{\partial^{2} w}{\partial t^{2}}=0
$$

Here $\rho$ and $E$ are density and Young's modulus of the beam's material while $A$ is the area and $I$ is the moment of inertia of the cross-section. With a rectangular cross-section we have $A=b h, I=A h^{2} / 12$, and the beam bending rigidity is given by

$$
D=E I / A=E h^{2} / 12 .
$$

For a time-harmonic excitation $w(x, t)=w(x, \omega) \mathrm{e}^{-\mathrm{i} \omega t}$ and the complex amplitude (spectrum) $w(x, \omega)$ is governed by the $1 \mathrm{D}$ equation

$$
\frac{\mathrm{d}^{4} w}{\mathrm{~d} x^{4}}-\kappa^{4} w=0
$$

in which $\kappa^{4}=\omega^{2} \rho / D$.

Since the patch action is modeled by the discrete moments (13), the bending moment $M(x)$ must be discontinuous at the points $x_{j}$ with jumps $M_{j}$ :

$$
\left.M\right|_{x=x_{j}^{-}}-\left.M\right|_{x=x_{j}^{+}}=(-1)^{j} M_{j}, \quad j=1, \ldots, 4 .
$$

Taking into account Eq. (16), this results in the conditions of compatibility put on the second derivative of $w$ :

$$
\left.w^{\prime \prime}\right|_{x=x_{j}^{-}}-\left.w^{\prime \prime}\right|_{x=x_{j}^{+}}=(-1)^{j+1} M_{j} / E I .
$$

All other derivatives are continuous due to the continuity of $w, \psi$ and $Q$, which should be met from the mechanics standpoint.

In this way, we arrive at a four-point ODE boundary-value problem for equation (18) with transition conditions

$$
\begin{aligned}
& w_{j}\left(x_{j}\right)-w_{j+1}\left(x_{j}\right)=0, \\
& w_{j}^{\prime}\left(x_{j}\right)-w_{j+1}^{\prime}\left(x_{j}\right)=0, \\
& w_{j}^{\prime \prime}\left(x_{j}\right)-w_{j+1}^{\prime \prime}\left(x_{j}\right)=b_{j}, \quad b_{j}=(-1)^{j+1} M_{j} / D A, \\
& w_{j}^{\prime \prime \prime}\left(x_{j}\right)-w_{j+1}^{\prime \prime \prime}\left(x_{j}\right)=0, \quad j=1, \ldots, 4 .
\end{aligned}
$$

Here $w_{m}(x), m=1, \ldots, 5$ are five parts of the function $w(x)$ related to the intervals $I, \ldots, V$ (Fig. 5) of the axis $x$, respectively. With patches of identical size $l_{0} \times b_{0} \times h_{0}$ the boundary points of the intervals are

$$
\begin{array}{ll}
x_{1}=-\left(l+l_{0}\right) / 2, & x_{3}=\left(l-l_{0}\right) / 2, \\
x_{2}=-\left(l-l_{0}\right) / 2, & x_{4}=\left(l+l_{0}\right) / 2 .
\end{array}
$$

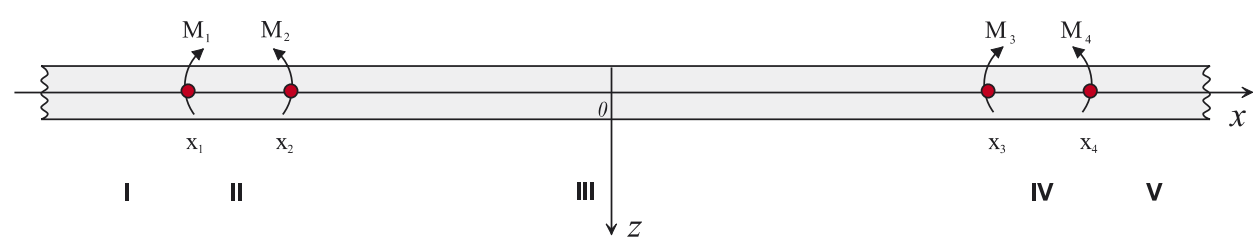

Fig. 5. A beam subjected to discrete bending moments. 
For the sake of simplifying further analysis, we suppose that the material properties of the patches, i.e. constants $E_{0}$ and $d_{31}$, are also identical. The case of unequal actuator sizes or properties only differs by values of $x_{j}$ and $b_{j}$ in Eq. (20).

The general solution of Eq. (18) in the intervals $I, \ldots, V$ has the form

$$
w_{m}(x)=c_{m, 1} \mathrm{e}^{\kappa x}+c_{m, 2} \mathrm{e}^{-\kappa x}+c_{m, 3} \mathrm{e}^{\mathrm{i} \kappa x}+c_{m, 4} \mathrm{e}^{-\mathrm{i} \kappa x}, \quad m=1, \ldots, 5
$$

with a total of 20 unknown constants $c_{m, j}$ to be defined from 16 coupling equalities (20). Four missing conditions come from the requirements imposed on $w(x)$ at infinity. It is natural to demand, first,

$$
w(x) \rightarrow 0 \quad \text { as } \quad|x| \rightarrow \infty
$$

and, second, $w(x)$ complies with Sommerfeld's condition: $w(x)$ must not contain terms associated with waves coming from infinity. The first of these limitations leads to $c_{1,2}=c_{5,1}=0$, while Sommerfeld's condition assumes that the terms with $\mathrm{e}^{\mathrm{i} \kappa x}$ and $\mathrm{e}^{-\mathrm{i} \kappa x}$ must be zero as $x<x_{1}$ and $x>x_{4}$, respectively; hence $c_{1,3}=c_{5,4}=0$.

Substitution of $w_{m}$ in the form (22) into the boundary conditions (20) results in a linear algebraic system

$$
\mathbf{A c}=\mathbf{b}
$$

with respect to the vector of unknown coefficients $\mathbf{c}=\left(\mathbf{c}_{1}, \mathbf{c}_{2}, \ldots, \mathbf{c}_{5}\right)$ of length 16 ; here $\mathbf{c}_{1}=\left(c_{1,2}, c_{1,4}\right)$, $\mathbf{c}_{5}=\left(c_{5,2}, c_{5,3}\right)$, and $\mathbf{c}_{m}=\left(c_{m, 1}, c_{m, 2}, c_{m, 3}, c_{m, 4}\right), m=2,3,4$. Due to the block structure of the matrix $\mathbf{A}$, system (22) can be solved explicitly. Using notations

$$
\begin{gathered}
e_{j 1}=\exp \left(-\kappa x_{j}\right), \quad e_{j 3}=\exp \left(-\mathrm{i} \kappa x_{j}\right), \\
e_{j 2}=\exp \left(\kappa x_{j}\right), \quad e_{j 4}=\exp \left(i \kappa x_{j}\right), \quad j=1, \ldots, 4,
\end{gathered}
$$

and $b_{j}$ defined in Eq. (20), the components of $\mathbf{c}_{m}$ can be written in the following form:

$$
\begin{aligned}
& c_{1,1}=\left(b_{1} e_{11}+b_{2} e_{21}+b_{3} e_{31}+b_{4} e_{41}\right) / 4 \kappa^{2}, \\
& c_{1,2}=c_{1,3}=0, \\
& c_{1,4}=\left(-b_{1} e_{14}-b_{2} e_{24}-b_{3} e_{34}-b_{4} e_{44}\right) / 4 \kappa^{2}, \\
& c_{2,1}=\left(b_{2} e_{21}+b_{3} e_{31}+b_{4} e_{41}\right) / 4 \kappa^{2}, \\
& c_{2,2}=-b_{1} e_{12} / 4 \kappa^{2}, \\
& c_{2,3}=b_{1} e_{13} / 4 \kappa^{2}, \\
& c_{2,4}=\left(-b_{2} e_{24}-b_{3} e_{34}-b_{4} e_{44}\right) / 4 \kappa^{2}, \\
& c_{3,1}=\left(b_{3} e_{31}+b_{4} e_{41}\right) / 4 \kappa^{2}, \\
& c_{3,2}=\left(-b_{1} e_{12}-b_{2} e_{22}\right) / 4 \kappa^{2}, \\
& c_{3,3}=\left(b_{1} e_{13}+b_{2} e_{23}\right) / 4 \kappa^{2}, \\
& c_{3,4}=\left(-b_{3} e_{34}-b_{4} e_{44}\right) / 4 \kappa^{2}, \\
& c_{4,1}=b_{4} e_{41} / 4 \kappa^{2}, \\
& c_{4,2}=\left(-b_{1} e_{12}-b_{2} e_{22}-b_{3} e_{32}\right) / 4 \kappa^{2}, \\
& c_{4,3}=\left(b_{1} e_{13}+b_{2} e_{23}+b_{3} e_{33}\right) / 4 \kappa^{2}, \\
& c_{4,4}=-b_{4} e_{44} / 4 \kappa^{2}, \\
& c_{5,1}=c_{5,4}=0, \\
& c_{5,2}=\left(-b_{1} e_{12}-b_{2} e_{22}-b_{3} e_{32}-b_{4} e_{42}\right) / 4 \kappa^{2}, \\
& c_{5,3}=\left(b_{1} e_{13}+b_{2} e_{23}+b_{3} e_{33}+b_{4} e_{43}\right) / 4 \kappa^{2} .
\end{aligned}
$$




\section{Layer model}

The explicit analytic representation (22), (25) and (26) for the complex amplitude $w(x, \omega)$ is a good reason to analyze traveling waves excited in a beam as a function of patch size $l_{0}$ and position $l$ as well as of the phase shift of the electric fields $\varphi_{n}$ and frequency $\omega$. However, $w(x)$ describes propagation of only the traveling bending wave associated with the terms $\mathrm{e}^{\mathrm{Fi} \kappa x}$ of the solution (22) in the intervals I and $\mathrm{V}$, respectively. The wavelength of this wave $\lambda_{b}=2 \pi / \kappa$. Since the beam equation was derived under the assumption $h \ll \lambda_{b}$, it puts certain limitations on the frequency band of applicability of this model. In practice excitation of an elastic waveguide at higher frequencies results in a much richer set of traveling modes than only one bending wave. To be able to estimate the limits of the beam model, we should compare it with a $2 \mathrm{D}$ model that allows for normal modes.

In the context of integral representations (8), (9), it can be any elastic laminate substructure. A difference in layer thickness and elastic properties results only in particular representations of Green's matrix $\mathbf{k}(\mathbf{x})$ and its Fourier symbol $\mathbf{K}(\alpha, z)$. Effective algorithms developed for $\mathbf{K}(\alpha, z)$ construction with an arbitrary stratification [7] allow us to treat $\mathbf{K}(\alpha, z)$ generally as a given matrix-function. In this paper we restrict ourselves to a homogeneous elastic layer of thickness $h$ : $|x|<\infty,-h \leqslant z \leqslant 0$, with stress-free sides $z=0$ and $-h$.

We should mention that similar integral equation approaches have been used in Refs. [8,9] for an elastic half-plane as a host structure.

With shear contact stresses $\mathbf{q}_{n}=\left(q_{n}, 0\right)$ produced by a pair of strips bonded to the surface $z=0$ at the intervals $\left[x_{1}, x_{2}\right]$ and $\left[x_{3}, x_{4}\right]$, representation (9) is reduced to the form

$$
\mathbf{u}(\mathbf{x})=\frac{1}{2 \pi} \int_{\Gamma} \mathbf{K}_{1}(\alpha, z) Q(\alpha) \mathrm{e}^{-\mathrm{i} \alpha x} \mathrm{~d} \alpha,
$$

where vector $\mathbf{K}_{1}=\left(K_{11}, K_{21}\right)$ is the first column of the original $2 \times 2$ matrix $\mathbf{K}$ and

$$
Q(\alpha)=\sum_{n=1}^{2} Q_{n}(\alpha), \quad Q_{n}(\alpha)=\int_{x_{2 n-1}}^{x_{2 n}} q_{n}(x) \mathrm{e}^{\mathrm{i} \alpha x} \mathrm{~d} \alpha .
$$

In the considered case the components of vector $\mathbf{K}_{1}$ can be written in a closed analytical form. In notations $[4,10]$

$$
\mathbf{K}_{1}=\frac{1}{\Delta(\alpha)}\left(\begin{array}{c}
-\mathrm{i} M_{1}(\alpha, z) \\
\alpha S_{1}(\alpha, z)
\end{array}\right)
$$

with

$$
\begin{aligned}
M_{1}(\alpha, z)= & -\mathrm{i} \sigma_{2}\left\{\alpha^{2}\left(\sigma_{1} \sigma_{2} \gamma^{2} \sinh \sigma_{2} z+\gamma^{4} \sinh \sigma_{1} z\right)\right. \\
& -\alpha^{2} \gamma^{4} \cosh \sigma_{2} h \sinh \sigma_{1}(z+h)+\alpha^{4} \sigma_{1} \sigma_{2} \sinh \sigma_{2} h \cosh \sigma_{1}(z+h) \\
& \left.-\alpha^{2} \sigma_{1} \sigma_{2} \gamma^{2} \cosh \sigma_{1} h \sinh \sigma_{2}(z+h)+\gamma^{6} \sinh \sigma_{1} h \cosh \sigma_{2}(z+h)\right\} \\
S_{1}(\alpha, z)= & -\mathrm{i}\left\{\sigma_{1} \sigma_{2}\left(\alpha^{2} \gamma^{2} \cosh \sigma_{2} z+\gamma^{4} \cosh \sigma_{1} z\right)\right. \\
& -\sigma_{1} \sigma_{2} \gamma^{4} \cosh \sigma_{2} h \cosh \sigma_{1}(z+h)+\alpha^{2} \sigma_{1}^{2} \sigma_{2}^{2} \sinh \sigma_{2} h \sinh \sigma_{1}(z+h) \\
& \left.-\alpha^{2} \sigma_{1} \sigma_{2} \gamma^{2} \cosh \sigma_{1} h \cosh \sigma_{2}(z+h)+\gamma^{6} \sinh \sigma_{1} h \sinh \sigma_{2}(z+h)\right\} \\
\Delta(\alpha)= & 2 \mu\left[-2 \alpha^{2} \sigma_{1} \sigma_{2} \gamma^{4}-\left(\gamma^{8}+\alpha^{4} \sigma_{1}^{2} \sigma_{2}^{2}\right) \sinh \sigma_{1} h \sinh \sigma_{2} h\right. \\
& \left.\quad+2 \alpha^{2} \sigma_{1} \sigma_{2} \gamma^{4} \cosh \sigma_{1} h \cosh \sigma_{2} h\right]
\end{aligned}
$$

where

$$
\gamma^{2}=\alpha^{2}-0.5 \kappa_{2}^{2}, \quad \sigma_{n}=\sqrt{\alpha^{2}-\kappa_{n}^{2}}, \quad n=1,2,
$$

$\kappa_{1}=\omega / v_{p}$ and $\kappa_{2}=\omega / v_{s}$ are the wave numbers of $P(n=1)$ and $S(n=2)$ body waves; $v_{p}$ and $v_{s}$ are their velocities. 
Integral Eq. (11) takes the form of two equations

$$
v_{n}(x)=\frac{1}{2 \pi} \sum_{m=1}^{2} \int_{\Gamma} K_{11}(\alpha) Q_{m}(\alpha) \mathrm{e}^{-\mathrm{i} \alpha x} \mathrm{~d} \alpha, \quad x_{2 n-1} \leqslant x \leqslant x_{2 n}, \quad n=1,2
$$

with the kernel symbol

$$
K_{11}(\alpha)=-\mathrm{i} M_{1}(\alpha, 0) / \Delta(\alpha)
$$

A numerical solution of Eq. (29) can be obtained by different ways, e.g. in line with the Galerkin scheme, using expansion of unknown $q_{n}(x)$ in terms of orthogonal polynomials or splines. We have been developing an approach involving reduction of the problem to an infinite algebraic system reasoning from the normal modes expansion (e.g. Ref. [11]). This method turned out to be most appropriate with the problem considered. A detailed description of its modification for the patches-layer contact problem is given in Ref. [12]. Here we will only present some numerical results obtained with this approach.

First of all, Figs. 6 and 7 give examples for a comparison between the beam and layer models. There are plots of the dimensionless displacement amplitude $|\bar{w}|=\left|w /\left(h e_{0}\left(1+v_{0}\right)\right)\right|, e_{0}=d_{31} E_{z}$ versus the dimensionless circular frequency $\omega=2 \pi f h / v_{s}$ ( $f$ is a dimensional frequency) at the points $x=0$, half-way between the strips (Fig. 6), and $x / h=50$, far away from the actuators (Fig. 7).

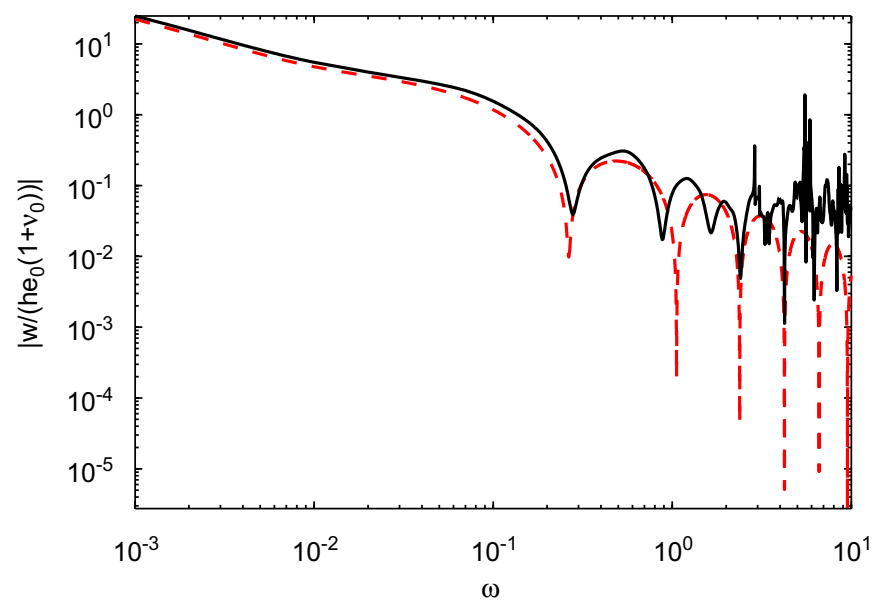

Fig. 6. Comparison of the beam model (dashed line) and the layer model (solid line); the amplitudes of the displacement at the half-way point between the strips $x=0$.

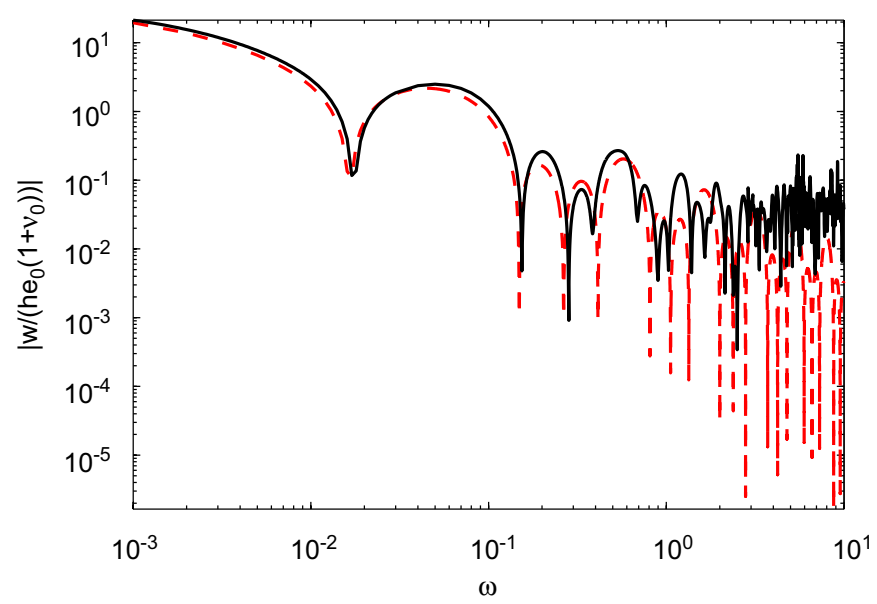

Fig. 7. The same at a point far away from the sources $x / h=50$. 
The plots are in a logarithmic scale. The calculations here and henceforward are carried out in a dimensionless form. For this purpose all variables are taken in the units expressed through three basic values: the beam or layer thickness $h$ as a unit of length, $S$-wave velocity $v_{s}$ in the beam (layer) as a unit of speed and the density $\rho$ as a unit of density. It leads to the following set of units:

$$
\begin{aligned}
& m_{0}=\rho h^{3} \text { for mass, } \\
& F_{0}=\rho h^{2} v_{s}^{2} \text { for force, } \\
& t_{0}=h / v_{s} \text { for time, } \\
& f_{0}=v_{s} / h \text { for frequency, }
\end{aligned}
$$

and so on.

Specifically, the plots in Figs. 6 and 7 are obtained with the following dimensionless input data:

Patches:

$l=16.667, l_{0}=8.333, h_{0}=0.1667, b_{0}=1.667, E_{0}=0.866, \rho_{0}=0.997,\left|e_{0}\right|=1, \varphi_{n}=0$.

Beam (layer):

$h=1, b=1.667(b=\infty), E=2.6, \rho=1\left(v_{s}=1\right.$, Poisson ratio $\left.v=0.3\right)$.

The plots show a very good agreement of beam and layer models at low frequencies $\omega<0.1$ and qualitatively different spectrums for $\omega>1$. In wavelengths $\lambda_{s}=2 \pi v_{s} / \omega$ and $\lambda_{b}=2 \pi\left(D /\left(\omega^{2} \rho\right)\right)^{1 / 4}$ for $S$ and bending waves, respectively, these inequalities can be read as follows: for $\lambda_{b}>13.6 h$ (or $\lambda_{s}>63 h$ ) the beam model works well while for $\lambda_{b}<4.8 h$ ( or $\lambda_{s}<6.3 h$ ) pure bending motion becomes too poor to describe all variety of normal modes excited.

We should note that in addition to the difference in the governing equations there exist more reasons for the result discrepancy. A first estimate shows that it can be due to the fact, that the contribution of the piezoceramics to the bending stiffnesses in sections II und IV is neglected. Another important reason lies in the approximation of a real stress distribution $q(x)$ by the discrete moments $M_{j}$. All these reasons yield an unremovable discrepancy even in statics, when $\omega=0$. Probably a difference in bending stiffness $D$ of the beam and a plate, into which the layer degenerates as $\omega \rightarrow 0$, also contributes into such a discrepancy. In the case under consideration $\left|w_{\text {beam }}\right| /\left|w_{\text {layer }}\right|=0.905$ as $\omega \rightarrow 0$ for both $x=0$ and $x / h=50$.

The agreement of $|w(x)|$ shapes exhibited by the two models considered is illustrated by Figs. $8-10$ plotted for $\omega=0.001,0.01$, and 0.1 . The markers on the plots show $\left|w_{\text {beam }}(x)\right|$ divided by the discrepancy coefficient 0.905 determined at $\omega=0$.

We can see once again that in the range of applicability the beam model provides quantitatively quite reasonable results in both frequency and space domains.

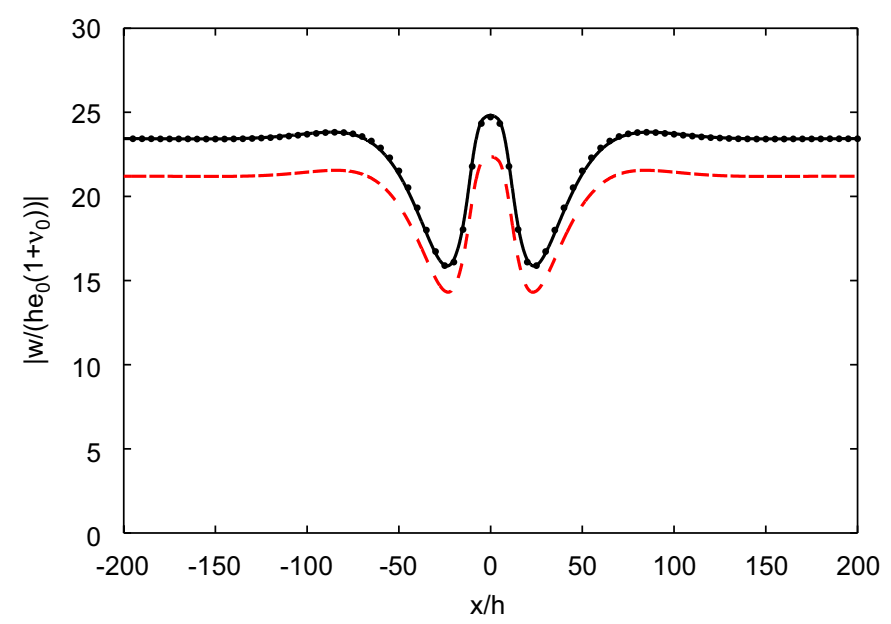

Fig. 8. The amplitude of the displacement for: layer model (solid line), beam model (dashed line) and beam model divided by the discrepancy coefficient 0.905 (dotted line); at $\omega=0.001$. 


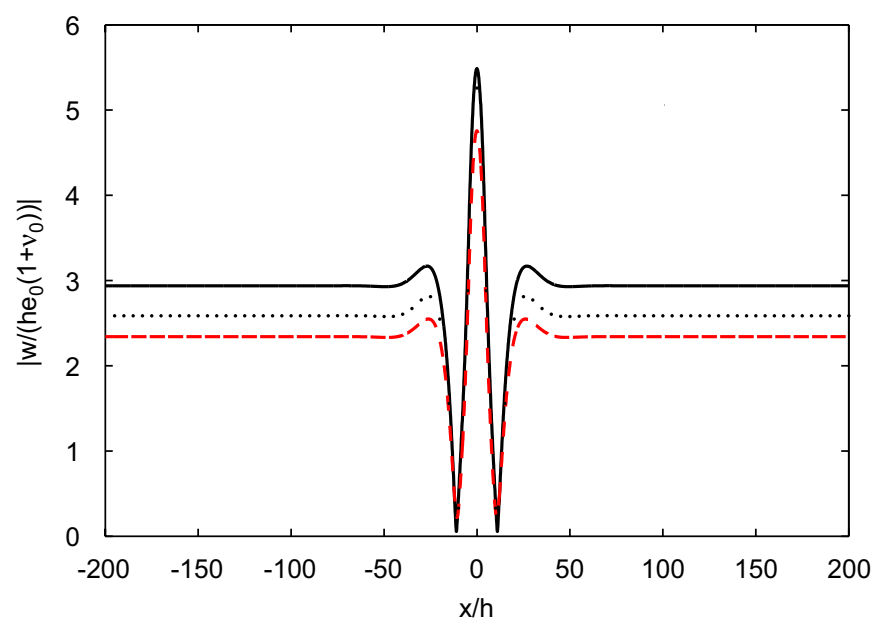

Fig. 9. The same at $\omega=0.01$.

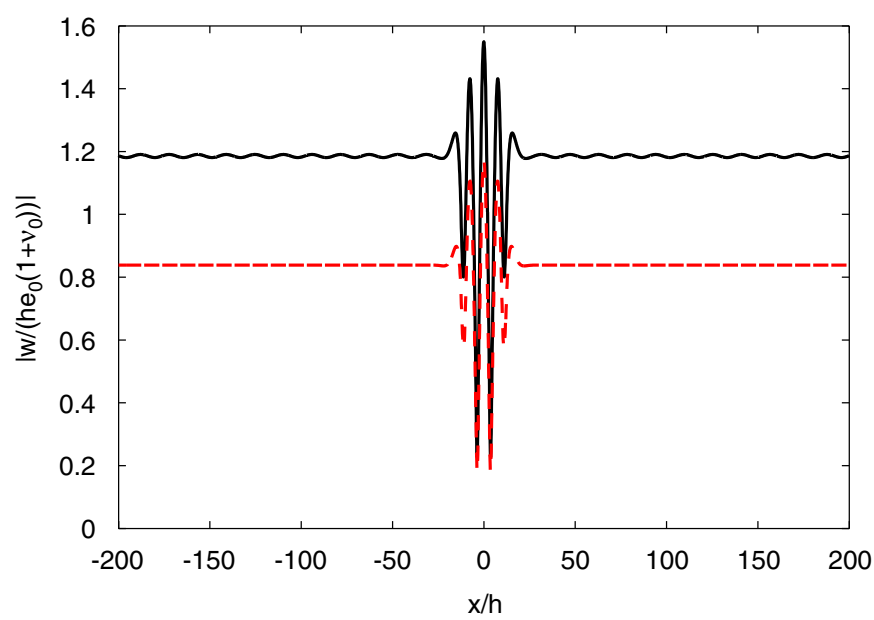

Fig. 10. The same at $\omega=0.1$.

\section{Wave analysis}

Thus, the beam solution derived analytically is a good tool for fast parametrical analysis of bending waves excited in a thin elastic waveguide by a pair of strip piezo-actuators. Having used this model, we intend to clarify the next questions:

How do the spacial distribution and the overall amplitude of the displacement $|w(x)|$ depend on

- the distance between the strips $l$,

- the phase shifts $\varphi_{n}$ of the two patches,

- the strip length $l_{1}$,

- the excitation frequency $\omega$,

- the ratio of $l$ or $l_{1}$ to the wavelength $\lambda_{b}$ ?

How do these parameters control the radiation of the wave energy in different directions?

Can a complete suppression of the radiation in one of two directions occur? 
Below we present selected plots exemplifying our attempts to clarify these questions. The models were calculated for the following dimensional parameters.

\section{Patches:}

$l_{0}=70 \mathrm{~mm}, h_{0}=1 \mathrm{~mm}, b_{0}=25 \mathrm{~mm}$, the material is PIC-141 with $\rho_{0}=7860 \mathrm{~kg} / \mathrm{m}^{3}, E_{0}=1 / 12.4 \times 10^{12} \mathrm{~Pa}$ and $v=0.3$.

\section{Beam:}

$h=3 \mathrm{~mm}, b=25 \mathrm{~mm}$, the material is steel ST-37 with $\rho=7850 \mathrm{~kg} / \mathrm{m}^{3}, v_{s}=3208 \mathrm{~m} / \mathrm{s}, E=2.1 \times 10^{11} \mathrm{~Pa}$, and $v=0.3$.

The dimensionless input data for this case are

Patches:

$l_{0}=23.333, h_{0}=0.333, b_{0}=8.333, \rho_{0}=0.9936, E_{0}=0.9983, v=0.3,\left|e_{0}\right|=1, \varphi_{n}=0$.

\section{Beam:}

$h=1, b=8.333, \rho=1, v_{s}=1, E=2.6$.

Fig. 11 displays the lengthwise distribution of the amplitude of oscillation $|w|$ (subplot 1) and of the real part of the complex amplitude $\operatorname{Re}\{w\}$ (subplot 2) at the dimensionless frequency $\omega=0.002(f=340 \mathrm{~Hz})$. The subplot 2 gives an instant snapshot of the beam's oscillating form. The distance between the patch edges $l_{1}=x_{3}-x_{2}$ (Fig. 3) is taken to be equal to the length of the bending wave $\lambda_{b} / h=96\left(\lambda_{b}=0.288 \mathrm{~m}\right)$. The curve of subplot 1 shows that minima of $|w|$ occur at the edges $x_{k}$, while the patch centers $x_{0,1}, x_{0,2}$ and the point $x=0$ are points of local maxima.

Apart from the sources $|w|$ became practically independent of $x$ (straight horizontal lines for $|x|>190$ in the subplot 1). However, the beam bends in that zone sinusoidally with the $\lambda_{b}$ period in accordance with propagation of the bending traveling wave. The curve of subplot 2 reminds this fact.

Fig. 12 illustrates the frequency effect on $|w(x)|$. The distance $l_{1}$ is chosen to be $l_{1}=\lambda_{b}$ again, but for a set of frequencies from Table 1 (subplots 1-6, respectively). These plots show that the change of frequency with the respective distance alteration keeps qualitatively the form of $|w(x)|$ curves, but their general level descends monotonically as $\omega$ grows and the strips come closer.

The plots of Fig. 13 demonstrate the effect of the actuator phase shifts. The dashed lines relate to the situation without shifts: $\varphi_{2}-\varphi_{1}=0$, while subplots $1-6$ show the amplitude of bending oscillations $|w(x)|$
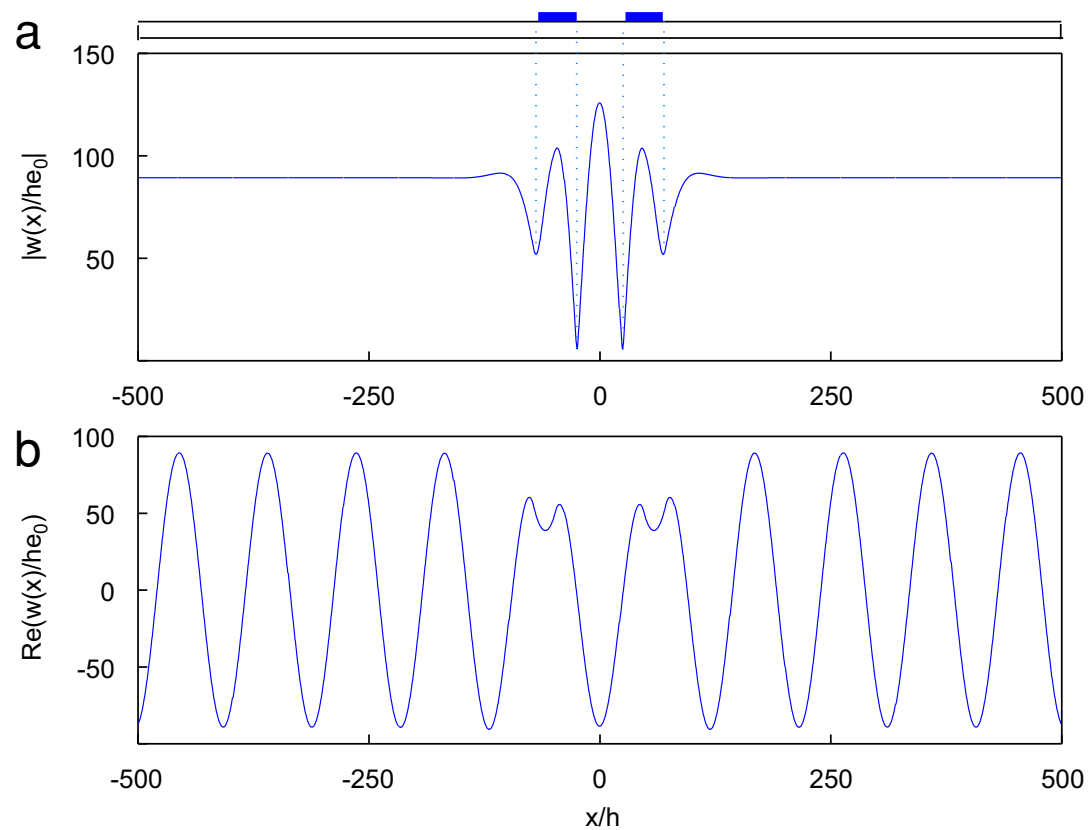

Fig. 11. The absolute value (a) and the corresponding real part (b) of the complex bending amplitude $w(x) ; \omega=0.002, l_{1}=x_{3}-x_{2}=\lambda_{b}$. 

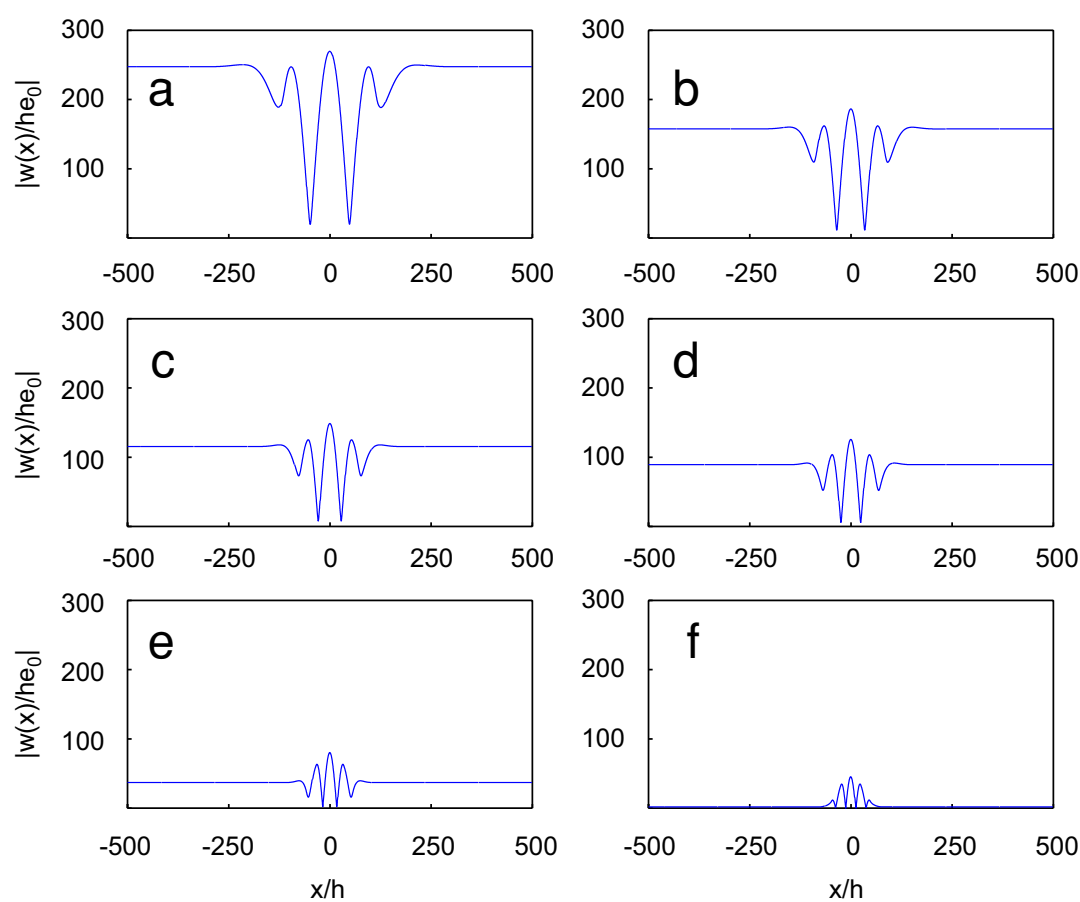

Fig. 12. The frequency effect: amplitude of the displacement $|w(x)|$ for $\omega=0.0005,0.001,0.0015,0.002,0.004,0.008$ (subplots (a)-(f)) for the distances $l_{1}=\lambda_{b}$ given in Table 1 .

Table 1

Frequencies and wavelengths in Fig. 12

\begin{tabular}{lrrrrrr}
\hline$\omega$ & 0.0005 & 0.001 & 0.0015 & 0.002 & 0.004 & 0.008 \\
\hline$f(\mathrm{~Hz})$ & 85 & 170 & 225 & 340 & 681 & 1362 \\
$\lambda_{b}(\mathrm{~m})$ & 0.575 & 0.407 & 0.332 & 0.288 & 0.203 & 0.144 \\
$\lambda_{b} / h$ & 192 & 136 & 111 & 96 & 68 & 48 \\
\hline
\end{tabular}

generated in the beam when $\varphi_{2}-\varphi_{1}=\pi / 6, \pi / 4, \pi / 3, \pi / 2, \pi$ and $3 \pi / 2$, respectively. The frequency is chosen as $\omega=0.002$ and the distance $l_{1}=\lambda_{b}$. One can see that the phase shift's increase from 0 to $\pi / 2$ results in a suppression of the radiation into the left direction down to almost complete damping when $\varphi_{1}=0, \varphi_{2}=\pi / 2$. In the latter, the amplitude of the radiation into the right hand side becomes approximately $\sqrt{2}$ times greater than with no shifts. That is, the wave energy doubles. From $\varphi_{2}=\pi / 2$ to $\varphi_{2}=\pi$ the amplitude of the traveling waves radiated in both directions is restored approximately to the same level as with no shifts. For shifts from $\pi$ to $2 \pi$ (or from $-\pi$ to 0 ) the plots are mirror images of the plots obtained for $0 \leqslant \varphi_{2} \leqslant \pi$ with the maximal suppression of the right traveling waves when $\varphi_{2}=3 \pi / 2$ (or $\varphi_{2}=-\pi / 2$ ).

To estimate the effect of the distance between the strips let us consider different ceramics allocations with $l_{1}=k \lambda_{b}, k=1 / 8,1 / 4,1 / 2,1,2,4$ (subplots $1-6$ of Fig. 14, respectively): $\omega=0.002, \varphi_{1}=\varphi_{2}=0$. One can see that strips separation results in number of local maxima and minima of $|w(x)|$ between the actuators, but it has almost no effect on the radiating waves except at the $\lambda_{b} / 4$ distance when an anti-resonance with radiation damping occurs.

Finally, Fig. 15 illustrates the effect of the actuator's size. The length of the patches $l_{0}=k \lambda_{b}$, $k=1,1 / 2,1 / 4,1 / 8,1 / 16$, and $1 / 32$ (subplots $1-6$ ). Thus, the ceramics are shrunk while the distance between the edges $l_{1}=x_{3}-x_{2}$ is kept constant and equal to $\lambda_{b} ; \omega=0.002$. 

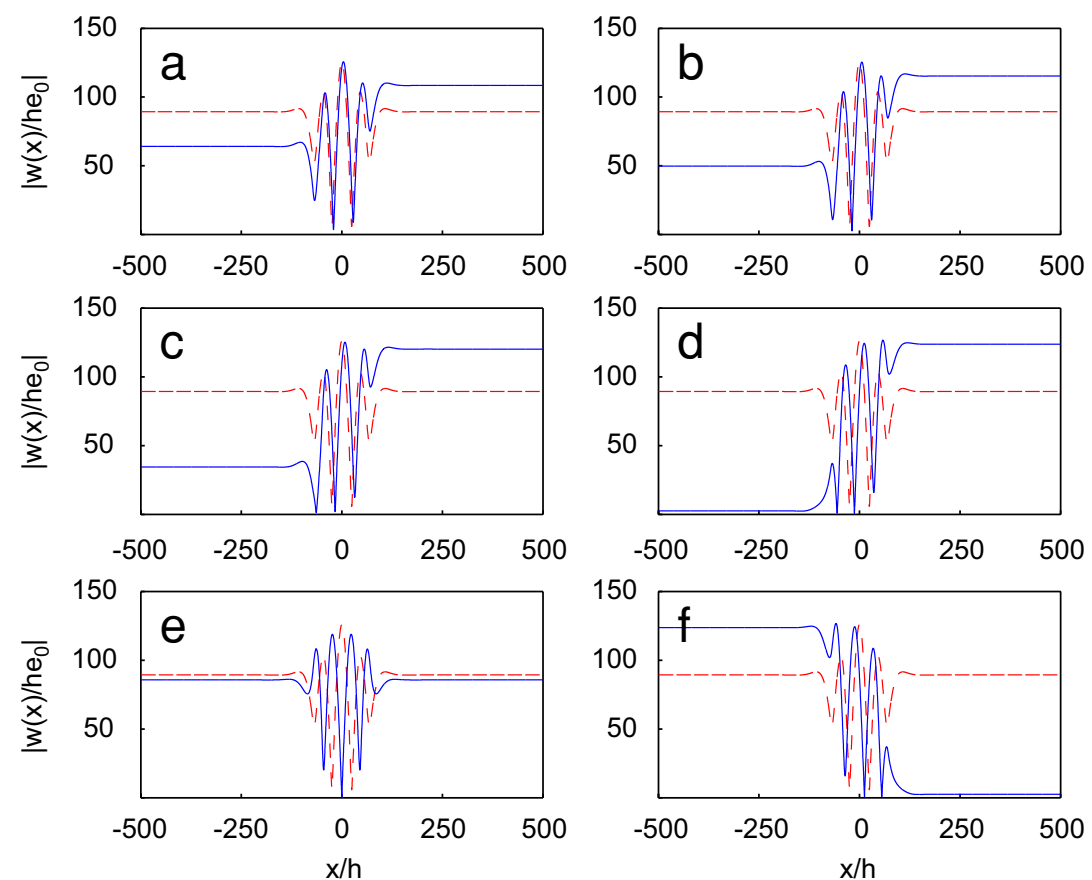

Fig. 13. The effect of the phase shifts: comparison of the amplitudes of the displacement $|w(x)|$ for a phase shift $\varphi=0$ (dashed line) with the amplitudes for phase shifts $\varphi_{2}=\pi / 6, \pi / 4, \pi / 3, \pi / 2, \pi, 3 \pi / 2$ (solid lines in subplots (a)-(f)).
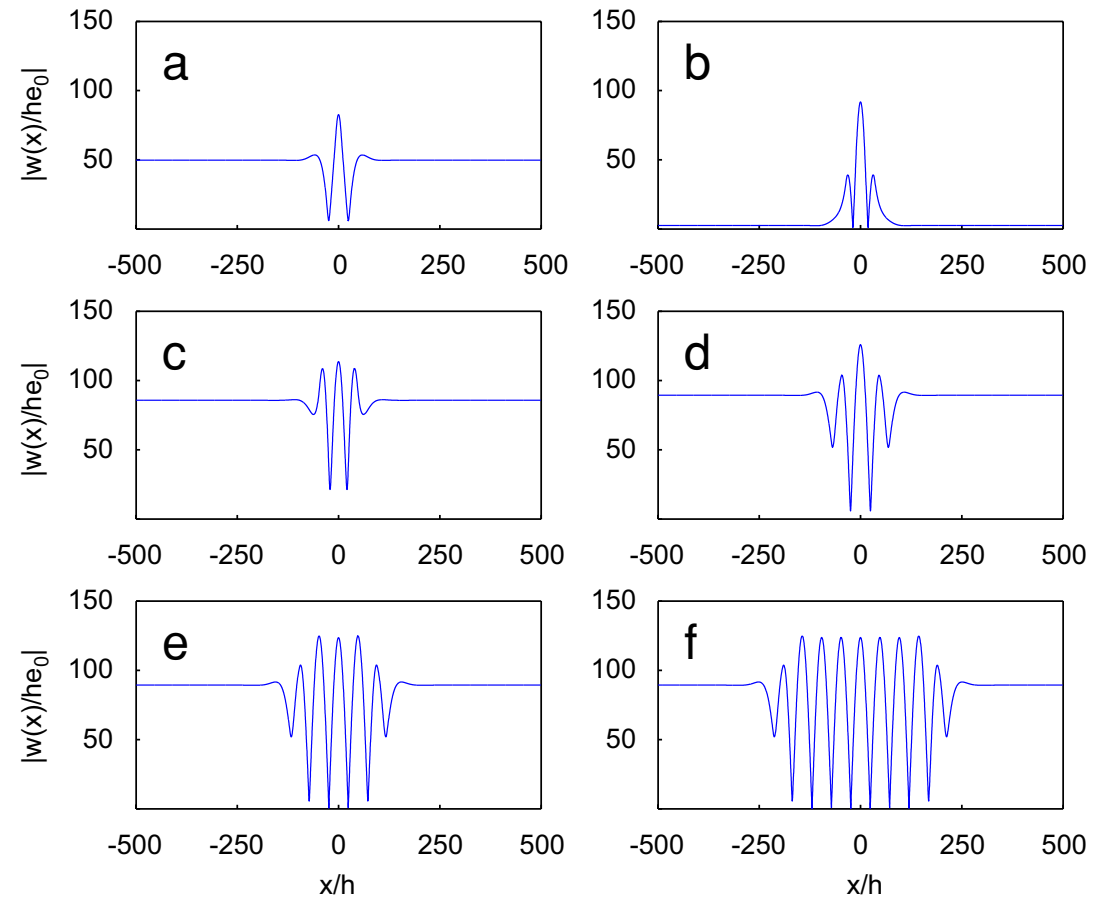

Fig. 14. The effect of the distance $l_{1}=x_{3}-x_{2}$ between the strips: amplitude of the displacement $|w(x)|$ for $l_{1}=k \lambda_{b}, k=$ $1 / 8,1 / 4,1 / 2,1,2,4$ (subplots (a)-(f)). 

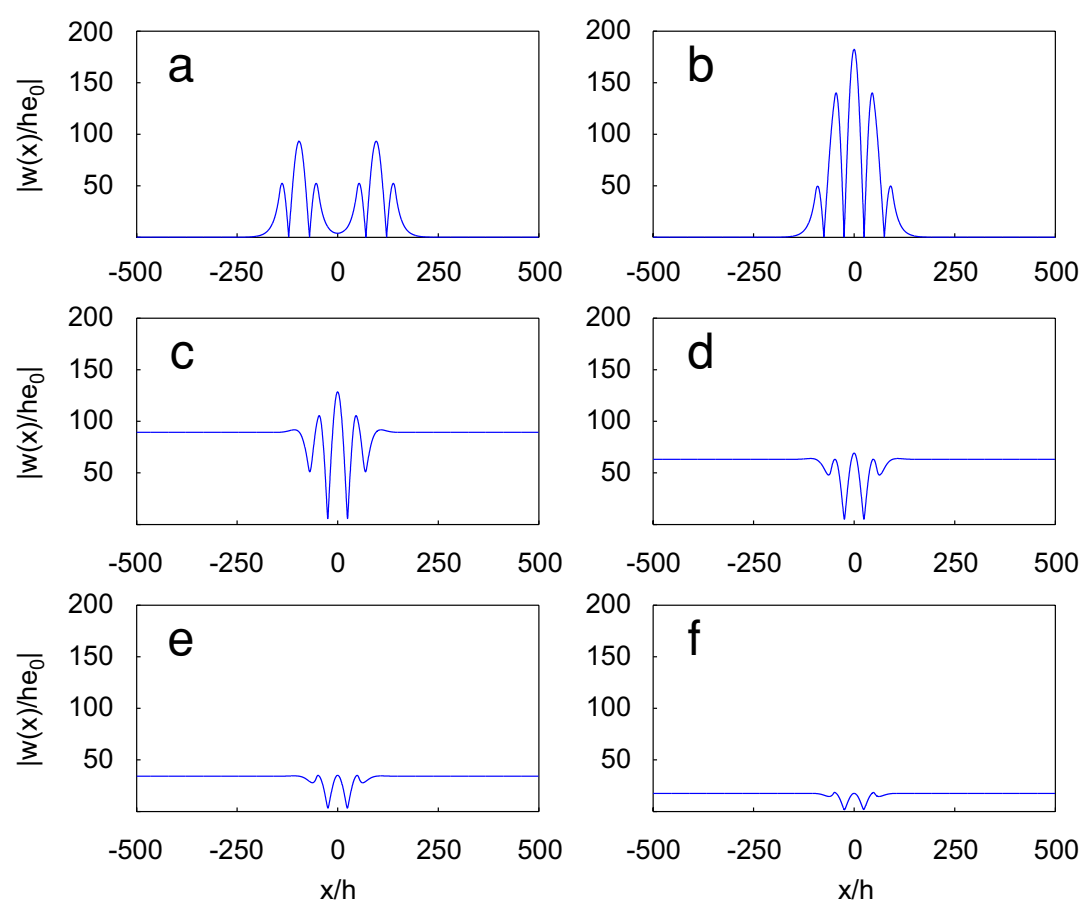

Fig. 15. The effect of the actuator's size $l_{0}=x_{2}-x_{1}=x_{4}-x_{3}$ : amplitude of the displacement $|w(x)|$ for $l_{0}=k \lambda_{b}, k=1,1 / 2,1 / 4$, $1 / 8,1 / 16,1 / 32$ (subplots (a)-(f)).

When the distance between the edges becomes comparable with $\lambda_{b}$, the patch shrinking results only in a monotonic decrease of radiation (subplots 3-6). This is quite natural, for the exciting forces induced in the patches are proportional to their volume. In contrast, when the patches are large and close to each other (subplots 1-2), the radiation is suppressed due to antiphase combination of waves generated by their different parts. This damping of traveling waves takes place in spite of high displacement amplitudes in the contact zone and between the sources.

\section{Summary}

We have presented a mathematical model for simulating harmonic bending waves excited in a thin elastic waveguide by electrically induced piezoceramic elements. The model enables fast parametric analysis of the effects of material properties, patch position and size, as well as of an inducing electric field, phase shifts, and frequency. The beam model has been verified numerically against a more complicated layer model, which gave in addition an assessment of the range of its validity.

\section{Acknowledgments}

The work has been supported by a research scholarship of the German Academic Exchange Service (DAAD) and by the Russian Foundation for Basic Research (RFBR) Grant 04-01-00801.

\section{References}

[1] B. Jaffe, W. Cook Jr., H. Jaffe, Piezoelectric Ceramics, Academic Press, New York, 1971.

[2] H.F. Tiersten, Linear Piezoelectric Plate Vibrations: Elements of Linear Theory of Piezoelectricity and the Vibrations of Piezoelectric Plates, Plenum Press, New York, 1969. 
[3] K. Ruschmeyer, Piezokeramik: Grundlagen Werkstoffe Applikationen, Renningen-Malmsheim, 1995.

[4] V.A. Babeshko, E.V. Glushkov, N.V. Glushkova, Energy vortices and backward fluxes in elastic waveguides, Wave Motion 16 (1992) 183-192.

[5] D. Gross, W. Hauger, E.H.W. Schnell, P. Wriggers, Technische Mechanik, Band 4: Hydromechanik, Elemente der Höheren Mechanik, Numerische Methoden, Springer-Lehrbuch, 1993.

[6] W. Seemann, A linear ultrasonic traveling wave motor of the ring type, Smart Materials and Structures 5 (1996) $361-368$.

[7] V.A. Babeshko, E.V. Glushkov, N.V. Glushkova, Methods of Green's matrix calculation for a stratified elastic half-space, Zhurnal Vychislitelnoy Matematiki i Matematicheskoy Fiziki 27 (1987) 93-101 (in Russian).

[8] B. Zhang, A. Boström, A.J. Niklasson, Antiplane shear waves from a piezoelectric strip actuator: exact versus effective boundary condition solutions, Smart Materials and Structures 13 (2004) 161-168.

[9] X.D. Wang, S.A. Meguid, On the electroelastic behaviour of a thin piezoelectric actuator attached to an infinite host structure, International Journal of Solids and Structures 37 (2000) 3231-3252.

[10] N.V. Glushkova, Determination and Accounting Singular Behavior in Elasticity, Dr Sc Thesis, University of Rostov-on-Don, 2000 (in Russian).

[11] E.V. Glushkov, N.V. Glushkova, Blocking property of energy vortices in elastic waveguides, Journal of Acoustical Society of America 102 (1997) 1356-1360.

[12] E.V. Glushkov, N.V. Glushkova, W. Seemann, O.V. Kvasha, Elastic wave excitation in a layer by piezoceramic patch actuators, Acoustical Physics 52 (2006) 398-407. 